Supplementary Information for

\title{
Disinfection byproducts in Rajasthan, India: Are trihalomethanes a sufficient indicator of disinfection byproduct exposure in low-income countries?
}

Kirin E. Furst ${ }^{1}$, Rachel M. Coyte ${ }^{2}$, Maggie Wood ${ }^{1}$, Avner Vengosh ${ }^{2}$, and William A. Mitch ${ }^{1}$

${ }^{1}$ Department of Civil and Environmental Engineering, Stanford University, 473 Via Ortega, Stanford, California 94305, United States

${ }^{2}$ Nicholas School of the Environment, Duke University, Durham, North Carolina 27708, United States

*Contact Information: email: wamitch@stanford.edu, Phone: 650-725-9298, Fax: 650-723-7058

1 Text

5 Figures

10 Tables

22 Pages 


\section{Table of Contents}

Table S1: International DBP regulations $\quad$ S3

$\begin{array}{ll}\text { Figure S1: Sample locations } & \text { S4 }\end{array}$

Table S2: Basic water quality parameters $\quad$ S6

Table S3: Chlorine residual data $\quad$ S8

Text S1: Artificial sweetener analytical method $\quad$ S9

Table S4: Artificial sweetener analytical parameters $\quad$ S10

Table S5: Artificial sweetener results $\quad$ S11

Table S6: Toxic potency metrics $\quad$ S12

Figure S2: DBP concentrations in Jaipur July $2017 \quad$ S13

Table S7: DBP concentrations in high-income countries $\quad$ S14

Table S8: Summary of DBP concentrations and comparisons to high-income countries S15

Table S9: Correlations between DBP concentrations and toxic potency-weighted DBPs S16

Figure S3: Correlations between THM4 and haloacetonitriles $\quad$ S17

Figure S4: Water quality parameter concentrations relevant to DBPs $\quad$ S18

Table S10: Correlations between artificial sweeteners and DBP classes $\quad$ S19

Figure S5: DBP formation from chloramine SDS treatment of select samples $\quad$ S20

$\begin{array}{ll}\text { References } & \text { S21 }\end{array}$ 
Table S1: International regulations and guidelines for disinfection byproduct concentrations $(\mu \mathrm{g} / \mathrm{L})$ in drinking water.

\begin{tabular}{lccccccc}
\hline \multicolumn{1}{c}{ DBP } & WHO & U.S. $^{2}$ & China $^{3}$ & India $^{4}$ & Mexico $^{5}$ & Iran $^{6}$ & Egypt $^{7}$ \\
\hline \hline Chloroform & 300 & 70 & 60 & 200 & --- & 300 & --- \\
Bromodichloromethane & 60 & 0 & 60 & 60 & --- & 60 & --- \\
Dibromochloromethane & 100 & 60 & 100 & 100 & --- & 100 & --- \\
Bromoform & 100 & 0 & 100 & 100 & --- & 100 & --- \\
THM4 & $1^{\mathrm{a}}$ & $80^{\mathrm{b}}$ & $1^{\mathrm{a}}$ & --- & 200 & $1^{\mathrm{a}}$ & 100 \\
Monochloroacetic acid & 20 & 70 & --- & --- & --- & --- & --- \\
Dichloroacetic acid & 50 & 0 & 50 & --- & --- & --- & --- \\
Trichloroacetic acid & 200 & 20 & 100 & --- & --- & --- & --- \\
Bromoacetic acid & --- & --- & --- & --- & --- & --- & --- \\
Dibromoacetic acid & --- & --- & --- & --- & --- & --- & --- \\
HAA5 & --- & $60^{\mathrm{b}}$ & --- & --- & --- & --- & --- \\
Trichloroacetonitrile & & & & --- & --- & --- & 1 \\
Dichloroacetonitrile & $20^{\mathrm{c}}$ & --- & --- & --- & --- & --- & 20 \\
Dibromoacetonitrile & 70 & --- & --- & --- & --- & --- & 70 \\
NDMA & 0.1 & --- & --- & --- & --- & --- & --- \\
\hline
\end{tabular}

${ }^{\text {a }}$ Maximum recommended result from WHO THM4 Guideline Value equation S1 (unitless):

$$
\frac{C_{T C M}}{G V_{T C M}}+\frac{C_{B D C M}}{G V_{B D C M}}+\frac{C_{D B C M}}{G V_{D B C M}}+\frac{C_{T B M}}{G V_{T B M}} \leq 1.0[\mathrm{~S} 1]
$$

where $C=$ concentration and $G V=$ guideline value

b EPA Maximum Contaminant Level; all other EPA values provided are non-enforceable Maximum Contaminant Level Goals.

${ }^{\mathrm{c}}$ Provisional guideline value (WHO, 2005)

References: ${ }^{1}$ WHO, 2004, 2005, 2011; ${ }^{2}$ US EPA, 1998; ${ }^{3}$ PRC, 2006; ${ }^{4}$ BIS, 2012; 5 MNWL, 1994; ${ }^{6}$ ISIRI, 2009; ${ }^{7} \mathrm{EMOH}, 2007$. 


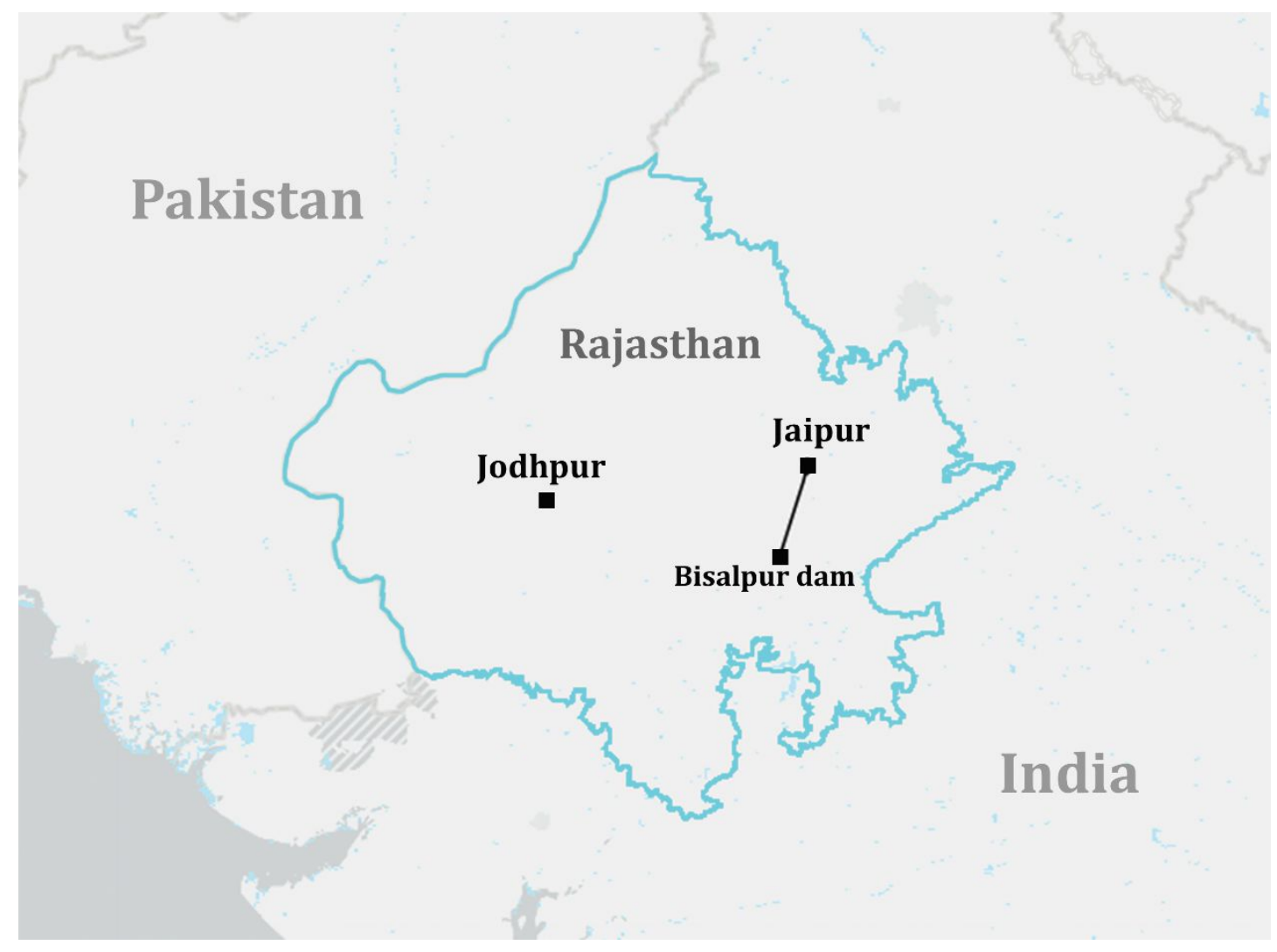

\section{Jodhpur}
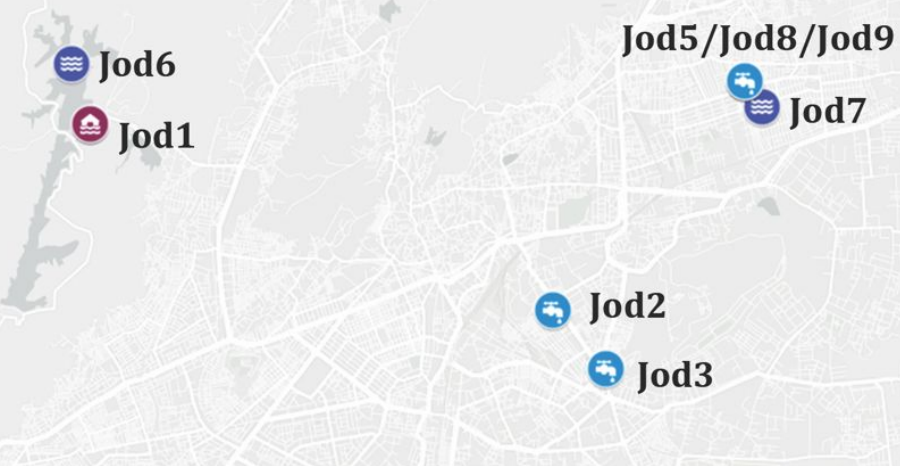

Jod5/Jod8/Jod9

을 $\operatorname{Jod} 7$

6 Jod2

6 Jod3

6 Jod4

Jod10

(2) Drinking water treatment plant

Е

5. Tap water sample location

き Untreated water sample location 


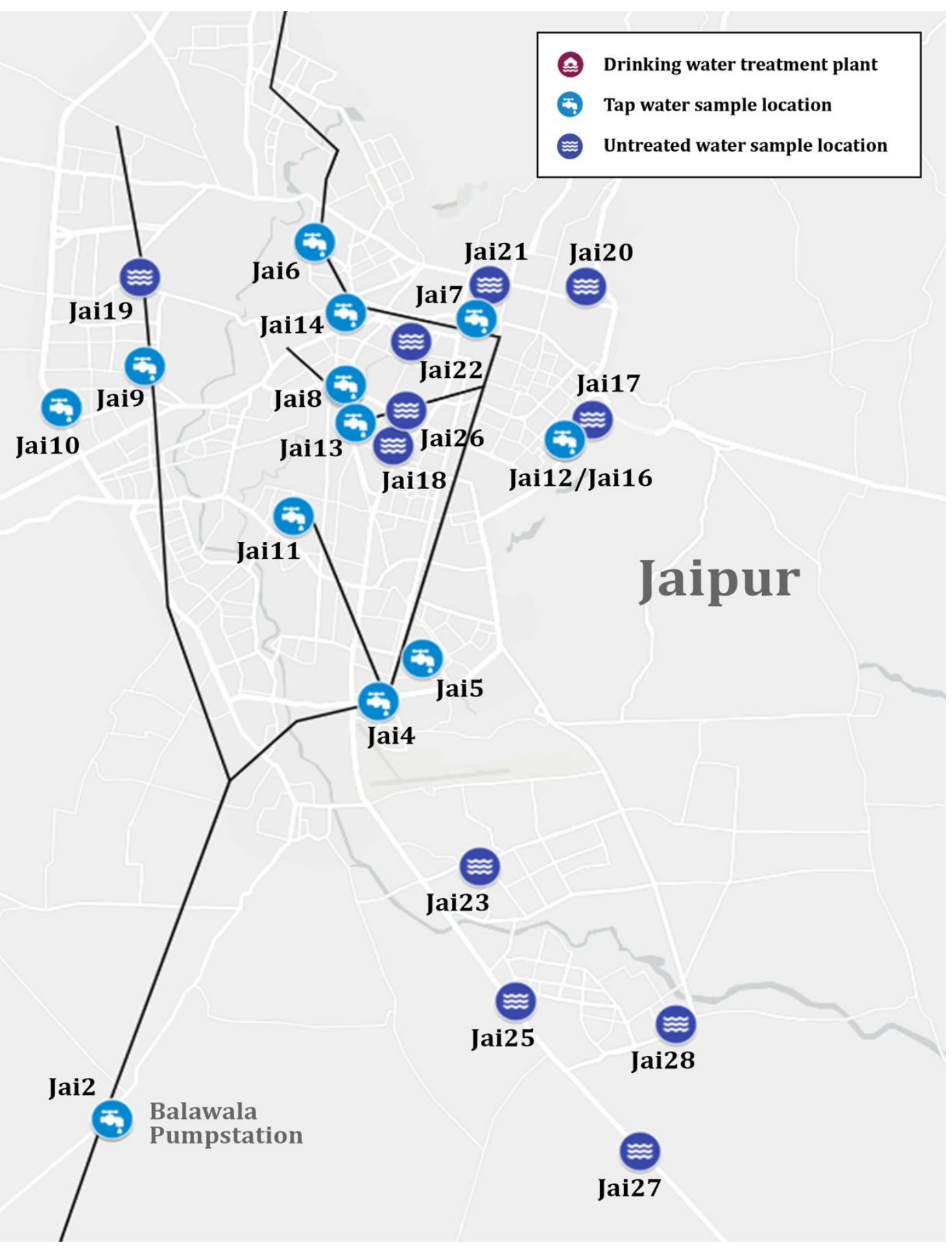

Figure S1: A) The study region of Rajasthan, with sampling locations in Jodhpur and Jaipur. In Jaipur, surface water is treated at the Bisalpur Dam before transmission to the city's distribution system. B) Jodhpur and C) Jaipur, with treated samples represented by light blue tap symbols and untreated samples represented by water lines (see legend). 
Table S2A: Sample information and basic water quality for tap and source waters from Jaipur

\begin{tabular}{|c|c|c|c|c|c|c|c|c|c|c|c|c|c|c|c|}
\hline Date & Name & $\begin{array}{c}\text { Original } \\
\text { name }\end{array}$ & Type & Source & Site & $\begin{array}{c}\text { Temp } \\
\text { C }\end{array}$ & $\mathbf{p H}$ & $\begin{array}{c}\text { Cond } \\
\mu \mathrm{S} / \mathrm{cm}^{3}\end{array}$ & $\begin{array}{l}\text { TDS } \\
\mathrm{mg} / \mathrm{L}\end{array}$ & $\begin{array}{l}\text { DOC } \\
\mathrm{mg} / \mathrm{L}\end{array}$ & $\begin{array}{c}\text { Bromide } \\
\text { mg/L }\end{array}$ & $\begin{array}{c}\begin{array}{l}\text { Iodine } \\
\text { (total) }\end{array} \\
\mathrm{mg} / \mathrm{L}\end{array}$ & $\begin{array}{c}\text { Nitrate } \\
\mathrm{mg} / \mathrm{L}\end{array}$ & $\begin{array}{l}\mathbf{U} \mathbf{V}_{254} \\
\mathrm{~cm}^{-1}\end{array}$ & $\begin{array}{c}\text { SUVA } \\
\mathrm{L} /\left(\mathrm{mg}^{*} \mathrm{~m}\right)\end{array}$ \\
\hline Dec-18 & jail & jai302 & tap & surface & water plant & 21 & 7.4 & 337 & 296 & 3.9 & 0.020 & --- & 0.15 & --- & --- \\
\hline Dec-18 & jai2 & jai303 & tap & surface & pumphouse & 22 & 7.6 & 348 & 291 & 3.4 & 0.010 & --- & 0.14 & --- & --- \\
\hline Dec-18 & jai3 & jai304 & tap & surface & pumphouse & 22 & 7.4 & 340 & 287 & 3.8 & 0.030 & --- & 0.13 & --- & --- \\
\hline Dec-18 & jai4 & jai305 & tap & surface & pumphouse & 23 & 7.5 & 345 & 286 & 3.7 & 0.020 & --- & 0.12 & --- & --- \\
\hline Dec-18 & jai5 & jai306 & tap & surface & pumphouse & 23 & 7.5 & 345 & 295 & 3.8 & 0.010 & --- & 1.31 & --- & --- \\
\hline Dec-18 & jai6 & jai307 & tap & surface & pumphouse & 20 & 7.8 & 356 & 293 & 3.3 & 0.020 & --- & 1.04 & --- & --- \\
\hline Dec-18 & jai7 & jai308 & tap & surface & pumphouse & 24 & --- & 335 & 289 & 3.7 & 0.010 & --- & 0.17 & --- & --- \\
\hline Dec-18 & jai8 & jai202 & tap & ground & household & --- & 7.7 & 1430 & 1110 & 3.1 & 0.185 & --- & 206 & --- & --- \\
\hline Jun-17 & jai9 & jai141 & tap & surface & household & 35 & 9.1 & 388 & 263 & 3.5 & 0.015 & 0.023 & 6.18 & --- & --- \\
\hline Jun-17 & jai10 & jai140 & tap & surface & business & 32 & 8.9 & 360 & 234 & 3.7 & 0.024 & 0.022 & 1.56 & --- & --- \\
\hline Jun-17 & jai11 & jai139 & tap & surface & pumphouse & 33 & 8.9 & 359 & 240 & 4.0 & 0.013 & 0.023 & 1.65 & --- & --- \\
\hline Jun-17 & jai12 & jai134 & tap & surface & pumphouse & 30 & 8.8 & 405 & 297 & 3.7 & 0.015 & 0.031 & 11.5 & --- & --- \\
\hline Jun-17 & jai13 & jai136 & tap & surface & & 30 & 8.9 & 394 & 233 & 5.0 & 0.022 & 0.027 & $<0.22$ & --- & --- \\
\hline Jun-17 & jai14 & jai137 & tap & surface & & 33 & 9.0 & 361 & 238 & 4.2 & 0.013 & 0.026 & 1.66 & 0.0439 & 1.1 \\
\hline Dec-18 & jai15 & surajpura & raw & surface & water plant & 22 & 7.9 & 356 & 306 & 4.7 & 0.140 & $0.000^{\mathrm{a}}$ & 0.05 & 0.0473 & 1.0 \\
\hline Jun-17 & jai16 & jai133 & raw & ground & tubewell & 29 & 8.1 & 774 & 540 & 3.0 & 0.090 & 0.054 & 93.0 & 0.0470 & 1.6 \\
\hline Jun-17 & jai17 & jai135 & raw & ground & tubewell & 32 & 7.7 & 1166 & 760 & 2.7 & 0.088 & 0.017 & 200 & 0.0519 & 1.9 \\
\hline Jun-17 & jai18 & jai138 & raw & ground & tubewell & 32 & 8.2 & 962 & 625 & 2.0 & 0.125 & 0.013 & 124 & 0.0351 & 1.8 \\
\hline Jun-17 & jai19 & jai142 & raw & ground & tubewell & 32 & 8.1 & 926 & 627 & 2.1 & 0.090 & 0.016 & 114 & 0.0484 & 2.3 \\
\hline Jun-17 & jai20 & $a b 23$ & raw & ground & tubewell & 32 & 7.7 & 1831 & 1210 & 2.8 & 0.186 & 0.016 & 170 & 0.0633 & 2.3 \\
\hline Jun-17 & jai21 & $24 r$ & raw & ground & tubewell & 29 & 8.1 & 1289 & 921 & 3.1 & 0.164 & 0.012 & 111 & 0.0361 & 1.2 \\
\hline Jun-17 & jai22 & $25 r$ & raw & ground & tubewell & 32 & 8.3 & 2490 & 1580 & 2.0 & 0.632 & 0.014 & 494 & 0.0613 & 3.1 \\
\hline Jun-17 & jai23 & $26 r$ & raw & ground & tubewell & 29 & 8.6 & 1079 & 827 & 4.0 & 0.279 & 0.062 & 20.1 & 0.0258 & 0.64 \\
\hline Jun-17 & jai24 & jai-hi & raw & ground & tubewell & 27 & 7.6 & 2750 & 1140 & 2.9 & 0.274 & 0.013 & 174 & 0.0514 & 1.8 \\
\hline Jan-18 & jai25 & jai203 & raw & ground & tubewell & 24 & 8.0 & 1663 & 1470 & 4.0 & 0.345 & 0.284 & 76.2 & 0.0218 & 0.55 \\
\hline Jan-18 & jai26 & jai201 & raw & ground & tubewell & 25 & 7.7 & 1062 & 787 & 2.8 & 0.480 & 0.070 & 183 & 0.0188 & 0.68 \\
\hline
\end{tabular}




\begin{tabular}{|c|c|c|c|c|c|c|c|c|c|c|c|c|c|c|c|}
\hline Jan-18 & jai27 & jai204 & raw & ground & tubewell & 27 & 7.1 & 3245 & 3120 & 2.4 & 3.696 & 0.227 & 45.7 & 0.0178 & 0.73 \\
\hline Jan-18 & jai28 & jai205 & raw & ground & tubewell & 26 & 7.1 & 1738 & 1720 & 4.9 & 0.626 & 0.702 & 55.9 & 0.0569 & 1.2 \\
\hline
\end{tabular}

a Halides for this sample were measured using ion chromatography, so this is the concentration of iodide instead of total iodine.

Table S2B: Sample information and basic water quality for tap and source samples from Jodhpur

\begin{tabular}{|c|c|c|c|c|c|c|c|c|c|c|c|c|c|c|c|}
\hline Date & Name & $\begin{array}{l}\text { Original } \\
\text { name }\end{array}$ & Type & Source & Site & $\begin{array}{c}\text { Temp } \\
\text { C }\end{array}$ & pH & $\begin{array}{l}\text { Cond } \\
\mu \mathrm{S} / \mathrm{cm}^{3}\end{array}$ & $\begin{array}{c}\text { TDS } \\
\mathrm{mg} / \mathrm{L}\end{array}$ & $\begin{array}{l}\text { DOC } \\
\mathrm{mg} / \mathrm{L}\end{array}$ & $\begin{array}{c}\text { Bromide } \\
\mathrm{mg} / \mathrm{L}\end{array}$ & $\begin{array}{c}\begin{array}{c}\text { Iodine } \\
\text { (total) }\end{array} \\
\text { mg/L }\end{array}$ & $\begin{array}{c}\text { Nitrate } \\
\mathrm{mg} / \mathrm{L}\end{array}$ & $\begin{array}{l}\mathbf{U} \mathbf{V}_{254} \\
\mathrm{~cm}^{-1}\end{array}$ & $\begin{array}{c}\text { SUVA } \\
\left(\mathrm{L} / \mathrm{mg}^{*} \mathrm{~m}\right)\end{array}$ \\
\hline Jan-18 & jod1 & jod208 & tap & surface & DWTP & 19 & 8.2 & 268 & 203 & 2.3 & 0.001 & --- & 4.29 & --- & --- \\
\hline Jan-18 & jod2 & jod202 & tap & surface & temple & --- & 8.1 & 288 & 208 & 2.6 & 0.014 & --- & 4.64 & --- & --- \\
\hline Jan-18 & jod3 & jod214 & tap & surface & temple & 19 & 8.3 & 261 & 200 & 2.5 & 0.003 & --- & 4.20 & --- & --- \\
\hline Jan-18 & jod4 & jod209 & tap & surface & temple & 21 & 8.3 & 264 & 203 & 2.4 & --- & --- & 3.94 & --- & --- \\
\hline Jan-18 & jod5 & jod205 & tap & mixed & temple & 18 & 8.4 & 350 & 265 & 2.6 & 0.017 & --- & 14.9 & --- & --- \\
\hline Jan-18 & jod6 & jod207 & raw & surface & reservoir & 18 & 8.1 & 255 & 195 & 2.0 & 0.008 & 0.024 & 3.51 & 0.0253 & 1.3 \\
\hline Jan-18 & jod7 & jod 203 & raw & ground & tubewell & 28 & 7.3 & 1578 & 1260 & 3.2 & 0.201 & 0.728 & 169 & 0.0272 & 0.85 \\
\hline Jan-18 & jod $8 *$ & jod204 & raw & ground & tubewell & 28 & 7.8 & 1550 & 1260 & 3.4 & 0.206 & 0.507 & 178 & 0.0232 & 0.69 \\
\hline Jan-18 & jod9* & jod206 & raw & ground & tubewell & 30 & 7.2 & 1600 & 1300 & 3.4 & 0.196 & 0.555 & 181 & 0.0294 & 0.87 \\
\hline Jan-18 & jod10 & jod215 & raw & ground & tubewell & 28 & 7.1 & 3675 & 2800 & 1.1 & 1.059 & 1.400 & 38.4 & 0.0869 & 7.7 \\
\hline
\end{tabular}

*Same tubewell site, different depths (30 $\mathrm{m}$ and $100 \mathrm{~m})$

Table S2 key: Temp = Temperature; Cond = Conductivity; TDS = Total Dissolved Solids; DOC = Dissolved Organic Carbon; SUVA = Specific UV Absorbance at $254 \mathrm{~nm}$. 
Table S3: Chlorine data for finished drinking water samples collected in Jaipur and Jodhpur

\begin{tabular}{lllcc}
\hline Name & $\begin{array}{c}\text { Original } \\
\text { name }\end{array}$ & Collection & \multicolumn{2}{c}{ Residual (mg/L as $\left.\mathbf{C l}_{2}\right)$} \\
method & Free & Total \\
\hline \hline jai1 & jai302 & Operator log & $\dagger 1.25$ & --- \\
jai2 & jai303 & Operator log & 0.7 & --- \\
jai3 & jai304 & Operator log & 2.0 & --- \\
jai4 & jai305 & Detector & 1 & --- \\
jai5 & jai306 & --- & --- & --- \\
jai6 & jai307 & --- & --- & --- \\
jai7 & jai308 & --- & --- & --- \\
jai8 & jai202 & --- & --- & --- \\
jai9 & jai141 & DPD & $<$ MRL & 0.14 \\
jai10 & jai140 & DPD & 0.73 & 1.2 \\
jai11 & jai139 & DPD & 0.71 & 0.92 \\
jai12 & jai134 & DPD & 0.28 & 0.70 \\
jai13 & jai136 & DPD & 0.87 & 1.1 \\
jai14 & jai137 & DPD & 1.2 & 1.5 \\
jod1 & jod208 & --- & --- & --- \\
jod2 & jod202 & DPD & non-detect & non-detect \\
jod3 & jod214 & DPD & slight & slight \\
jod4 & jod209 & --- & --- & --- \\
jod5 & jod205 & --- & --- & --- \\
\hline & & & &
\end{tabular}

Table key: DPD: Residuals were measured using a Hach Pocket Colorimeter II spectrophotometer (Hach, Loveland, $\mathrm{CO}$ ) with free and total chlorine DPD packets (MRL $0.1 \mathrm{mg}-\mathrm{Cl}_{2} / \mathrm{L}$ ).

${ }^{\dagger}$ Final chlorine dose applied, as recorded from the Surajpura utility information board.

${ }^{\ddagger}$ Residuals measured using the DPD method in Jodhpur with qualitative color reference scale. Non-detect $=$ no observable color change; slight $=$ minimal color change. 
Text S1: Additional methods/materials for artificial sweetener analysis

Source water samples were spiked with $50 \mu \mathrm{g} / \mathrm{L}$ deuterated sucralose-d6, and extracted onto Oasis HLB (200 g, $6 \mathrm{~mL}$ ) solid phase extraction (SPE) cartridges. The SPE cartridges were eluted with $6 \mathrm{~mL}$ of methanol, which was evaporated to $0.5 \mathrm{~mL}$ under a gentle stream of nitrogen gas while submersed in a $35{ }^{\circ} \mathrm{C}$ water bath. Methanol extracts were analyzed by liquid chromatography-mass spectrometry using an Agilent 1260 Infinity HPLC system coupled to an Agilent 6460 triple quadripole mass spectrometer (LC/MS/MS). Chromatographic separation was achieved with a Phenomenex Synergi $4 \mu \mathrm{m}$ Hydro-RP $(150 \times 3 \mathrm{~mm})$ column using acetonitrile and a $0.1 \%$ acetic acid aqueous solution as mobile phases. Analytes were quantified using electrospray ionization in the negative ion mode and the multiple reaction monitoring (MRM) mode. Parameters may be found in Table S4 below. Method detection limits were $\sim 10$ $\mathrm{ng} / \mathrm{L}$ for both sucralose and saccharin. 
Table S4: LC-MS-MS parameters used for analysis of the artificial sweeteners sucralose and saccharin

Parameters
Gas temperature
Gas flow
Nebulizer
Sheath gas temperature
Sheath gas flow
Capillary voltage
Nozzle voltage
Cell acceleration voltage

Compound-specific

Quantification ion

Confirmation ion

Fragmentation voltage

Collision energies

$\begin{array}{ll}\text { Setting } & \text { Units } \\ 250 & \mathrm{C} \\ 10 & \mathrm{~L} / \mathrm{min} \\ 45 & \mathrm{psi} \\ 350 & \mathrm{C} \\ 11 & \mathrm{~L} / \mathrm{min} \\ -3500 & \mathrm{~V} \\ 1500 & \mathrm{~V} \\ 2 & \mathrm{~V}\end{array}$

\begin{tabular}{llllll} 
Sucralose & & \multicolumn{2}{l}{ Sucralose-d6 } & \multicolumn{2}{c}{ Saccharin } \\
precursor & product & $\begin{array}{l}\text { precursor } \\
\text { product }\end{array}$ & precursor & product \\
395 & 35 & 401 & 35 & 182 & 42 \\
397 & 35 & 403 & 35 & 182 & 105.9 \\
150 & $\mathrm{~V}$ & 150 & $\mathrm{~V}$ & 123 & $\mathrm{~V}$ \\
15 & & 15 & & 24 &
\end{tabular}


Table S5: Artificial sweeteners measured in untreated drinking water samples from Jaipur and Jodhpur

\begin{tabular}{llrr}
\hline Name & Original name & $\begin{array}{c}\text { Saccharin } \\
\mathrm{ng} / \mathrm{L}\end{array}$ & $\begin{array}{c}\text { Sucralose } \\
\mathrm{ng} / \mathrm{L}\end{array}$ \\
\hline \hline jai14 & Surajpura & 16.8 & 31.3 \\
jai25 & jai203 & 13.6 & 69.2 \\
jai26 & jai201 & 61.1 & $<10$ \\
jai27 & jai204 & $<10$ & $<10$ \\
jai28 & jai205 & $<10$ & 286 \\
jod6 & jod207 & $<10$ & 31.9 \\
jod7 & jod203 & $<10$ & 88.1 \\
jod8 & jod204 & 26.7 & 63.6 \\
jod9 & jod206 & $<10$ & 68.5 \\
jod10 & jod215 & 28.6 & 52.7 \\
jod11 & jod211 & 19.8 & $<10$ \\
\hline
\end{tabular}


Table S6: Toxic potency values for disinfection byproducts measured in this study

\begin{tabular}{|c|c|c|c|}
\hline \multicolumn{2}{|c|}{ Disinfection byproduct } & $\operatorname{LECR}_{50}(M)^{a}$ & $\mathrm{LC}_{50}(\mathrm{M})^{\mathrm{b}}$ \\
\hline TCM & Trichloromethane & --- & $9.17 \times 10^{-3}$ \\
\hline BDCM & Bromodichloromethane & $1.69 \times 10^{-3}$ & $1.15 \times 10^{-2}$ \\
\hline DBCM & Dibromochloromethane & $1.00 \times 10^{-3}$ & $5.36 \times 10^{-3}$ \\
\hline TBM & Tribromomethane & --- & $3.96 \times 10^{-3}$ \\
\hline CDIM & Chlorodiiodomethane & NA & $2.41 \times 10^{-3}$ \\
\hline TIM & Triiodomethane & NA & $6.60 \times 10^{-5}$ \\
\hline DBIM & Dibromoiodomethane & NA & $1.91 \times 10^{-3}$ \\
\hline BCIM & Bromochloroiodomethane & NA & $2.42 \times 10^{-3}$ \\
\hline BDIM & Bromodiiodomethane & NA & $1.40 \times 10^{-3}$ \\
\hline DCIM & Dichloroiodomethane & NA & $4.13 \times 10^{-3}$ \\
\hline TBAL & Tribromoacetaldehyde & NA & $3.56 \times 10^{-6}$ \\
\hline TCAL & Trichloroacetaldehyde & NA & $1.16 \times 10^{-3}$ \\
\hline DBCAL & Dibromochloroacetaldehyde & NA & $5.15 \times 10^{-6}$ \\
\hline BDCAL & Bromodichloroacetaldehyde & NA & $2.04 \times 10^{-5}$ \\
\hline DBAN & Dibromoacetonitrile & NA & $2.85 \times 10^{-6}$ \\
\hline DCAN & Dichloroacetonitrile & NA & $5.73 \times 10^{-5}$ \\
\hline BCAN & Bromochloroacetonitrile & NA & $8.46 \times 10^{-6}$ \\
\hline TCAN & Trichloroacetonitrile & NA & $1.60 \times 10^{-4}$ \\
\hline DCAM & Dichloroacetamide & NA & $1.92 \times 10^{-3}$ \\
\hline BCAM & Bromochloroacetamide & NA & $1.71 \times 10^{-5}$ \\
\hline DBAM & Dibromoacetamide & NA & $1.22 \times 10^{-5}$ \\
\hline TCAM & Trichloroacetamide & NA & $2.05 \times 10^{-3}$ \\
\hline 1,1-DCP & 1,1-Dichloropropanone & NA & NA \\
\hline $1,1,1-\mathrm{TCP}$ & 1,1,1-Trichloropropanone & NA & NA \\
\hline TCNM & Trichloronitromethane & NA & $5.36 \times 10^{-4}$ \\
\hline
\end{tabular}

Table key: NA = Not Available.

${ }^{a} \mathrm{LECR}_{50}$ calculated using the age-adjusted $10^{-6}$ lifetime excess cancer risk level values from the IRIS database (UEPA 2019b). The LECR $_{50}$ was only used for weighting by toxic potency when it was found to be a more conservative estimate of toxic potency than the available $\mathrm{LC}_{50} .{ }^{\mathrm{b}}$ Wagner and Plewa, 2017. 

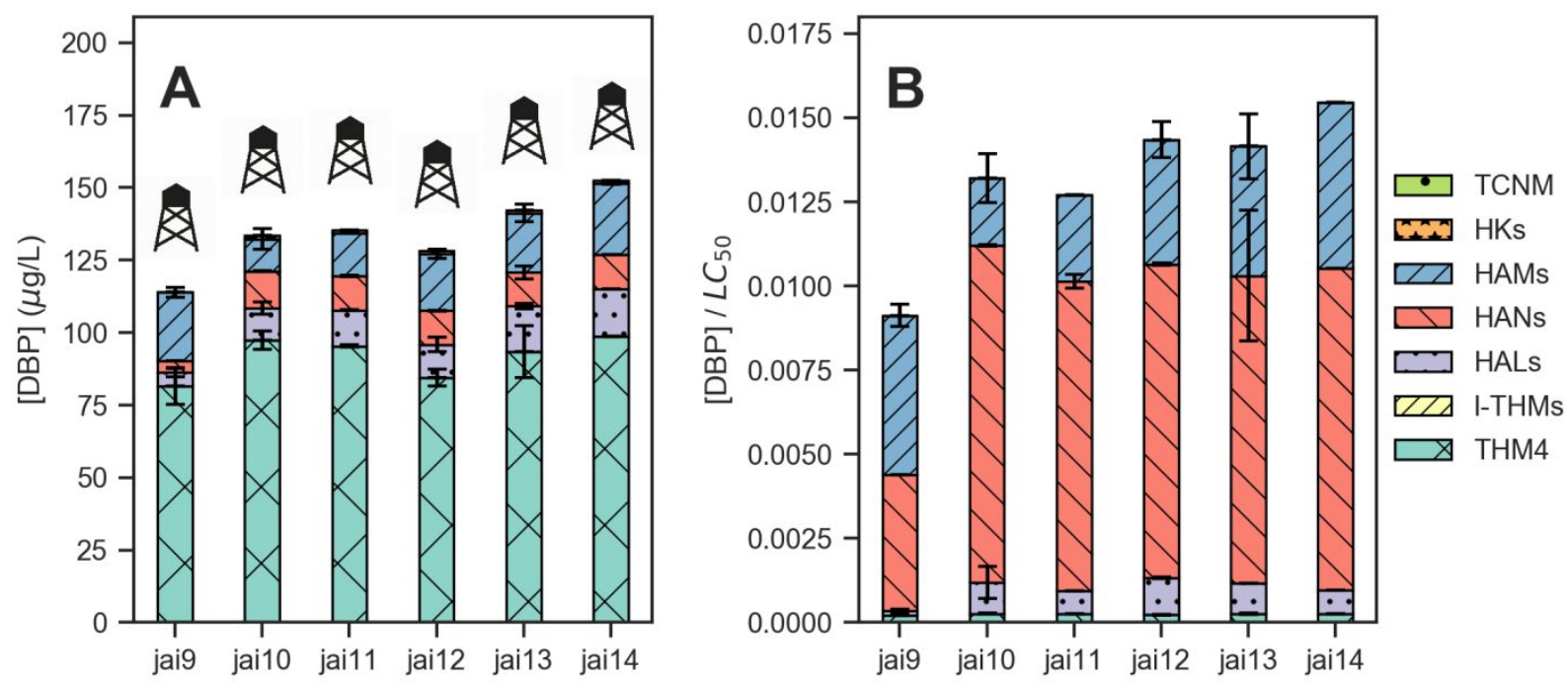

Figure S2: Jaipur July 2017 distribution system sample DBP concentrations (A) by weight and (B) by toxic potency. Tank symbols indicate samples collected from distribution system storage tanks. Error bars reflect the standard deviation of triplicate analytical measurements. SW = surface water-associated; GW = groundwater-influenced; TCNM = trichloronitromethane; HKs = haloketones; HAMs = haloacetamides; HANs = haloacetonitriles; HALs = haloacetaldehydes; I-THMs = iodinated THMs; THM4 = regulated THMs. 
Table S7: Reported volatile disinfection byproduct concentrations in select countries

\begin{tabular}{|c|c|c|c|c|c|c|c|c|c|c|c|c|c|c|c|c|c|c|c|}
\hline $\begin{array}{c}\mu g / L \\
\text { percentile }\end{array}$ & $\begin{array}{c}\text { THM4 } \\
50\end{array}$ & 75 & 90 & 100 & HAN & 75 & 90 & 100 & HALs & 75 & 90 & 100 & HAM & 75 & 90 & 100 & $\begin{array}{l}\text { Sample } \\
\text { type }\end{array}$ & Study context & Citation \\
\hline \multirow{5}{*}{ US } & 39 & 50 & - & 190 & 4.0 & - & - & 13 & - & - & - & - & - & - & - & - & Eff & $\begin{array}{l}35 \text { DTPs (data for winter } \\
\text { quarter) }\end{array}$ & Krasner 1989 \\
\hline & 34 & 56 & 81 & 320 & 2.9 & 5.3 & 8.3 & 52 & 2.8 & 6.5 & 11 & 92 & - & - & - & - & DS & $\begin{array}{l}\text { ICR survey; } 437+\text { DTPs, } \\
\sim 6300-6800 \text { DS sites }{ }^{\mathrm{b}, \mathrm{e}}\end{array}$ & USEPA 1998 \\
\hline & 28 & - & 64 & 210 & - & - & - & - & - & - & - & - & 0.6 & - & - & 2.8 & SDS & 12 large DTPs & Weinberg 2002 \\
\hline & $\begin{array}{c}31 \\
-\end{array}$ & $\begin{array}{c}45 \\
-\end{array}$ & $\begin{array}{l}- \\
-\end{array}$ & $\begin{array}{c}160 \\
-\end{array}$ & $\begin{array}{l}3.0 \\
-\end{array}$ & $\begin{array}{c}4.0 \\
-\end{array}$ & - & $\begin{array}{c}14 \\
-\end{array}$ & $\begin{array}{l}4.0 \\
-\end{array}$ & $\begin{array}{l}7.0 \\
-\end{array}$ & - & $\begin{array}{c}20 \\
-\end{array}$ & $\begin{array}{c}1.4 \\
-\end{array}$ & $\begin{array}{c}2.5 \\
-\end{array}$ & $\begin{array}{l}- \\
-\end{array}$ & $\begin{array}{l}7.4 \\
14\end{array}$ & $\begin{array}{c}\text { Eff } \\
\text { SDS }\end{array}$ & $\begin{array}{l}12 \text { DTPs, selected for } \\
\text { high bromide \& DOC }\end{array}$ & Krasner 2006 \\
\hline & - & - & - & - & - & - & - & - & 4.5 & - & - & 17 & - & - & - & - & Eff & & Jeong 2015 \\
\hline Canada & $\begin{array}{l}22 \\
34\end{array}$ & & & $\begin{array}{l}220 \\
340\end{array}$ & 1.9 & & & 16 & $\begin{array}{l}2.5 \\
4.8\end{array}$ & & & 23 & & & & & DS & $\begin{array}{l}35 \text { DTPs, values for } \\
\text { winter and summer }{ }^{\mathrm{a}, \mathrm{b}}\end{array}$ & Williams 1997 \\
\hline England & 20 & - & - & - & 2.8 & 4.2 & - & 12 & - & - & - & - & 1.4 & 2.1 & - & 7.0 & DS & 20 public water systems ${ }^{c}$ & Bond 2015 \\
\hline \multirow{2}{*}{ Spain } & 71 & - & - & 130 & 5.7 & - & - & 8.3 & $<1$ & - & & 12 & - & - & - & - & DS & 11 provinces & \multirow[t]{2}{*}{ Villaneuva 2012} \\
\hline & 14 & - & - & 44 & - & - & - & - & - & - & - & - & - & - & - & - & Eff & Barcelona (16 sites) & \\
\hline China & - & - & - & 510 & - & - & - & - & - & - & - & - & - & - & - & - & Eff & 15 DTPs in Beijing & Wei 2010 \\
\hline \multirow{2}{*}{ India } & - & - & - & 510 & - & - & - & - & - & - & - & - & - & - & - & - & Eff & 5 DTPs in Eastern India & Kumari 2015 \\
\hline & - & - & - & 590 & - & - & - & - & - & - & - & - & - & - & - & - & Eff & 8 DTPs in Eastern India & Mishra 2012 \\
\hline
\end{tabular}

Table key: DTP = drinking water treatment plant; Eff = finished plant effluent; DS = distribution system; SDS = simulated distribution system All reported values were rounded to 2 significant figures.

Measured all species as in this study, unless indicated otherwise:

a. DCAN only

b. TCAL only

c. DCAM, DBAM, and TCAM only (no BCAM)

d. CAM, BAM, DCAM, TCAM, DBAM (no BCAM)

e. The 1998 ICR Database (AUX1) was queried to acquire data for all sample events, and all subsequent analyses were conducted in Python (3.7). Sample events where concentrations were not measured for all THMs or for all HANs were excluded from analyses of those classes. For sample events where analyte concentrations were below the reporting limit, the value "-999" was replaced by 0 . Reported quantiles were calculated from concentrations of THM4 ( $\mathrm{n}=6826)$, HAN4 ( $\mathrm{n}=6308)$, and TCAL $(\mathrm{n}=6307)$ measured in distribution system samples (AVG1, AVG2, MAX) without aggregation. 
Table S8: Summary of results for finished tap waters and SDS-treated samples from Jaipur and Jodhpur, with literature values for conventional drinking waters and reuse waters in high-income countries.

\begin{tabular}{|c|c|c|c|c|c|c|c|c|c|c|c|}
\hline \multirow{2}{*}{ dataset } & \multirow{2}{*}{ category } & \multirow{2}{*}{ count } & \multicolumn{3}{|c|}{$\begin{array}{l}\text { cumulative toxic potency- } \\
\text { weighted concentration }\end{array}$} & \multicolumn{3}{|c|}{ THM4 ( $\mu \mathrm{g} / \mathrm{L})$} & \multicolumn{3}{|c|}{ haloacetonitriles $(\mu \mathrm{g} / \mathrm{L})$} \\
\hline & & & $\min$ & $50 \%$ & $\max$ & $\min$ & $50 \%$ & $\max$ & $\min$ & $50 \%$ & $\max$ \\
\hline \multirow[t]{4}{*}{ Jaipur $^{\mathrm{a}}$} & SW tap & 12 & $9.1 \mathrm{E}-03$ & $1.5 \mathrm{E}-02$ & $1.9 \mathrm{E}-02$ & 68.3 & 85.2 & 98.7 & 3.93 & 10.9 & 12.7 \\
\hline & SW SDS & 1 & $9.4 \mathrm{E}-03$ & --- & --- & 74.6 & --- & --- & 6.98 & --- & --- \\
\hline & GW tap & 1 & $2.7 \mathrm{E}-03$ & --- & --- & 16.6 & --- & --- & 1.09 & --- & --- \\
\hline & GW SDS & 13 & $7.6 \mathrm{E}-04$ & $2.2 \mathrm{E}-03$ & $8.1 \mathrm{E}-03$ & 17.0 & 32.2 & 59.3 & 0.39 & 1.20 & 4.66 \\
\hline \multirow[t]{4}{*}{ Jodhpur ${ }^{\mathrm{a}}$} & SW tap & 3 & $6.5 \mathrm{E}-04$ & $8.5 \mathrm{E}-04$ & $1.3 \mathrm{E}-03$ & 34.7 & 36.4 & 37.0 & 2.18 & 2.39 & 3.56 \\
\hline & SW SDS & 1 & $1.7 \mathrm{E}-03$ & --- & --- & 113 & --- & --- & 5.29 & --- & --- \\
\hline & Mix tap & 1 & $5.8 \mathrm{E}-05$ & --- & --- & 17.4 & --- & --- & 0.18 & --- & --- \\
\hline & GW SDS & 4 & $6.1 \mathrm{E}-03$ & 7.1E-03 & $5.1 \mathrm{E}-02$ & 31.5 & 38.0 & 211 & 3.61 & 4.25 & 29.7 \\
\hline \multirow[t]{3}{*}{ Chuang $2019^{\mathrm{b}}$} & conventional & 21 & $1.8 \mathrm{E}-05$ & $8.5 \mathrm{E}-04$ & $4.4 \mathrm{E}-03$ & 0.71 & 15.0 & 172.3 & 0.00 & 1.16 & 5.53 \\
\hline & reuse $\mathrm{O}_{3}$-BAC & 27 & $3.7 \mathrm{E}-05$ & $1.7 \mathrm{E}-03$ & $1.1 \mathrm{E}-02$ & 2.00 & 5.47 & 75.0 & 0.00 & 1.93 & 6.68 \\
\hline & reuse $\mathrm{RO}-\mathrm{AOP}$ & 5 & $6.0 \mathrm{E}-06$ & 4.1E-05 & $5.2 \mathrm{E}-04$ & 0.83 & 1.08 & 3.57 & 0.00 & 0.00 & 0.25 \\
\hline Krasner $2006^{c}$ & conventional & 12 & $2.5 \mathrm{E}-05$ & $1.5 \mathrm{E}-03$ & $5.0 \mathrm{E}-03$ & 8.40 & 29.7 & 83.0 & 0.00 & 1.50 & 7.60 \\
\hline Zeng $2016^{\mathrm{d}}$ & reuse $\mathrm{RO}-\mathrm{AOP}$ & 8 & $0.0 \mathrm{E}+00$ & 4.7E-04 & $9.0 \mathrm{E}-04$ & 0.00 & 9.64 & 17.9 & 0.00 & 1.72 & 3.73 \\
\hline Furst $2018^{\mathrm{e}}$ & reuse non-potable & 16 & $2.8 \mathrm{E}-03$ & $1.1 \mathrm{E}-02$ & $2.5 \mathrm{E}-02$ & 45.3 & 75.6 & 84.0 & 4.55 & 9.85 & 20.6 \\
\hline
\end{tabular}

Table key: DTP = drinking water treatment plant; conventional = conventional drinking water; $\mathrm{O}_{3}$ - $\mathrm{BAC}=$ potable reuse train centered on ozone and biological activated carbon treatment; $\mathrm{RO}-\mathrm{AOP}=$ reverse osmosis/advanced oxidation process treatment

${ }^{a}$ SDS-treated samples for Jaipur and Jodhpur include only samples targeting $1 \mathrm{mg} / \mathrm{L}$ as $\mathrm{Cl}_{2}$, in keeping with Figure 2 and the discussion in the manuscript. Jodhpur Mix tap is Jod5, appears to have been mixed with groundwater. Jodhpur and Jaipur SW tap statistics both exclude the treatment plant effluent samples.

b6 DTPs in several high-income nations

${ }^{\mathrm{c}} 12$ U.S. DTPs

d 5 treatment trains

e U.S. tertiary-treated municipal wastewater, chlorine-chloramine disinfection with and without addition of ammonia in the influent ammonia 
Table S9: Pearson correlations between concentrations of DBPs on a weight-basis and the cumulative toxic potency-weighted concentrations in SDS-treated samples.*

\begin{tabular}{r|rcc|rcc}
\hline $\begin{array}{r}\text { DBP family } \\
(\mu \mathrm{g} / \mathrm{L})\end{array}$ & \multicolumn{3}{|c|}{ Low FC residual } & \multicolumn{3}{c}{ High FC residual } \\
& $\mathbf{n}$ & $\mathbf{r}$ & $\mathbf{p}$ & $\mathbf{n}$ & $\mathbf{r}$ & $\mathbf{p}$ \\
\hline THM4 & 20 & 0.18 & $4.5 \mathrm{E}-01$ & 16 & 0.16 & $5.5 \mathrm{E}-01$ \\
I-THMs & 18 & -0.10 & $6.7 \mathrm{E}-01$ & 12 & -0.05 & $8.5 \mathrm{E}-01$ \\
HALs & 11 & 0.01 & $9.8 \mathrm{E}-01$ & 14 & -0.05 & $8.5 \mathrm{E}-01$ \\
HKs & 6 & 0.16 & $4.9 \mathrm{E}-01$ & 8 & -0.22 & $4.0 \mathrm{E}-01$ \\
HANs & 20 & 0.79 & $2.9 \mathrm{E}-05$ & 15 & 0.88 & $5.6 \mathrm{E}-06$ \\
HAMs & 12 & 0.13 & $5.7 \mathrm{E}-01$ & 7 & 0.03 & $9.0 \mathrm{E}-01$ \\
TCNM & 2 & -0.28 & $2.4 \mathrm{E}-01$ & 2 & -0.24 & $3.7 \mathrm{E}-01$ \\
\hline
\end{tabular}

Table key: n: number of samples with non-zero values; r: Pearson's correlation coefficient; $\mathrm{p}$ : $\mathrm{p}$ value for Pearson's correlation; Low FC residual = samples with a free chlorine residual of approximately $1 \mathrm{mg} / \mathrm{L}$ as $\mathrm{Cl}_{2}$ after 24 hours $\left(\mathrm{n}=20\right.$, range $0.58-1.7 \mathrm{mg} / \mathrm{L}$ as $\left.\mathrm{Cl}_{2}\right)$; High $\mathrm{FC}$ residual $=$ samples with a free chlorine residual near 3 or $4 \mathrm{mg} / \mathrm{L}$ as $\mathrm{Cl}_{2}$ after 24 hours $(\mathrm{n}=16$, range $2.7-4.1 \mathrm{mg} / \mathrm{L}$ as $\mathrm{Cl}_{2}$ ).

*Outlier jod10 was excluded from all calculations. 

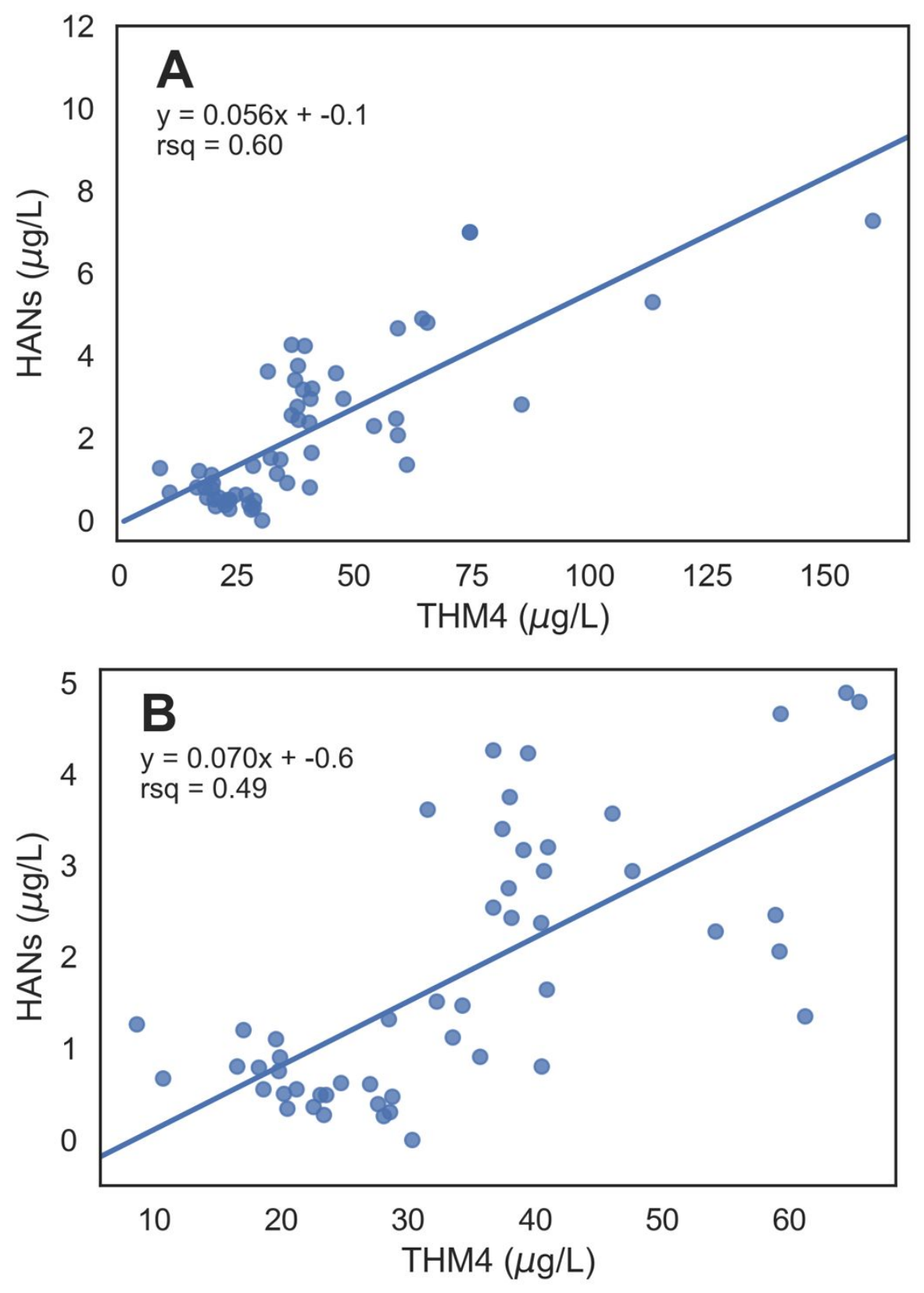

Figure S3: Scatterplots with line of best fit calculated by least squares linear regression for SDS samples treated with free chlorine for A) all sites, all doses and B) all groundwater sites, all doses (both exclude outlier Jod10). The r-squared (rsq) value is the Pearson correlation coefficient squared, which describes the proportion of the variance of the dependent variable that is explained by the independent variable. 

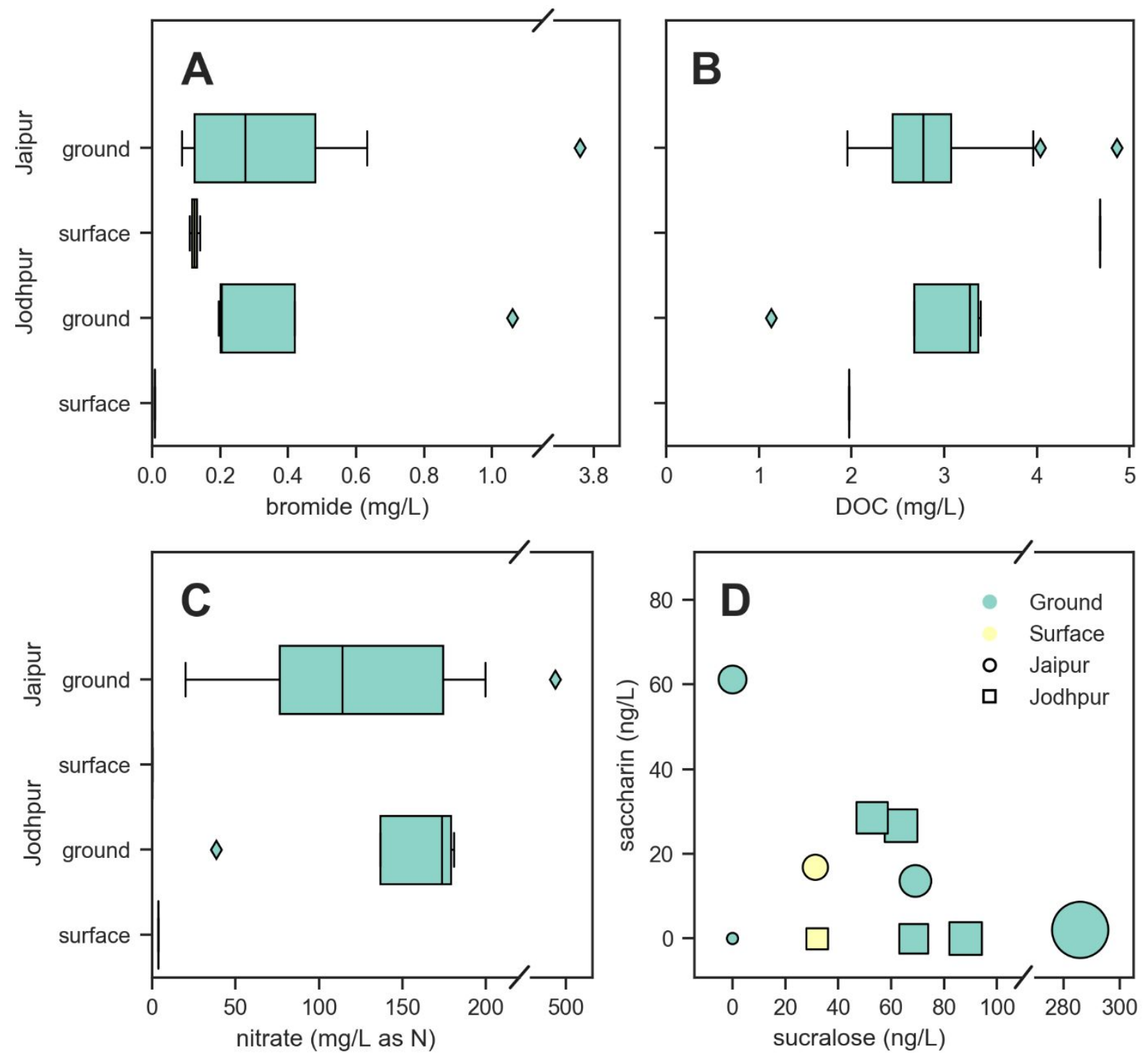

Figure S4: (A) Bromide, (B) DOC, and (C) nitrate concentrations in all untreated drinking water sources from Jaipur and Jodhpur. (D) Concentrations of sucralose and saccharin in 11 untreated samples from Jaipur and Jodhpur. Marker size represents nitrate concentration. 
Table S10: Pearson correlations between the concentrations of the artificial sweeteners sucralose and saccharin measured in untreated source waters and concentrations of DBP classes measured in source waters following SDS treatment with free chlorine.*

\begin{tabular}{r|ccc|ccc}
\hline $\begin{array}{r}\text { DBP family } \\
(\mu \mathrm{g} / \mathrm{L})\end{array}$ & \multicolumn{3}{|c|}{ Sucralose $(\mathrm{ng} / \mathrm{L})$} & \multicolumn{3}{c}{ Saccharin $(\mathrm{ng} / \mathrm{L})$} \\
& $\mathbf{r}$ & $\mathbf{p}$ & $\mathbf{n}$ & $\mathbf{r}$ & $\mathbf{p}$ \\
\hline \hline THM4 & 9 & 0.09 & $8.3 \mathrm{E}-01$ & 9 & -0.46 & $2.2 \mathrm{E}-01$ \\
I-THMs & 7 & 0.61 & $8.3 \mathrm{E}-02$ & 7 & -0.36 & $3.4 \mathrm{E}-01$ \\
HALs & 6 & -0.17 & $6.5 \mathrm{E}-01$ & 6 & -0.24 & $5.3 \mathrm{E}-01$ \\
HKs & 4 & -0.10 & $8.0 \mathrm{E}-01$ & 4 & -0.22 & $5.7 \mathrm{E}-01$ \\
HANs & 9 & 0.24 & $5.4 \mathrm{E}-01$ & 9 & -0.54 & $1.3 \mathrm{E}-01$ \\
HAMs & 6 & -0.23 & $5.6 \mathrm{E}-01$ & 6 & -0.46 & $2.1 \mathrm{E}-01$ \\
TCNM & 1 & -0.17 & $6.6 \mathrm{E}-01$ & 1 & -0.24 & $5.3 \mathrm{E}-01$ \\
\hline
\end{tabular}

Table key: n: number of samples with non-zero values for both the artificial sweetener and DBP class; r: Pearson's correlation coefficient; $p$ : p-value for Pearson's correlation.

*Samples with a free chlorine residual of approximately $1 \mathrm{mg} / \mathrm{L}$ as $\mathrm{Cl}_{2}$ after 24 hours, excluding outlier Jod10 ( $\mathrm{n}=9$, range $0.58-1.7 \mathrm{mg} / \mathrm{L}$ as $\left.\mathrm{Cl}_{2}\right)$. 

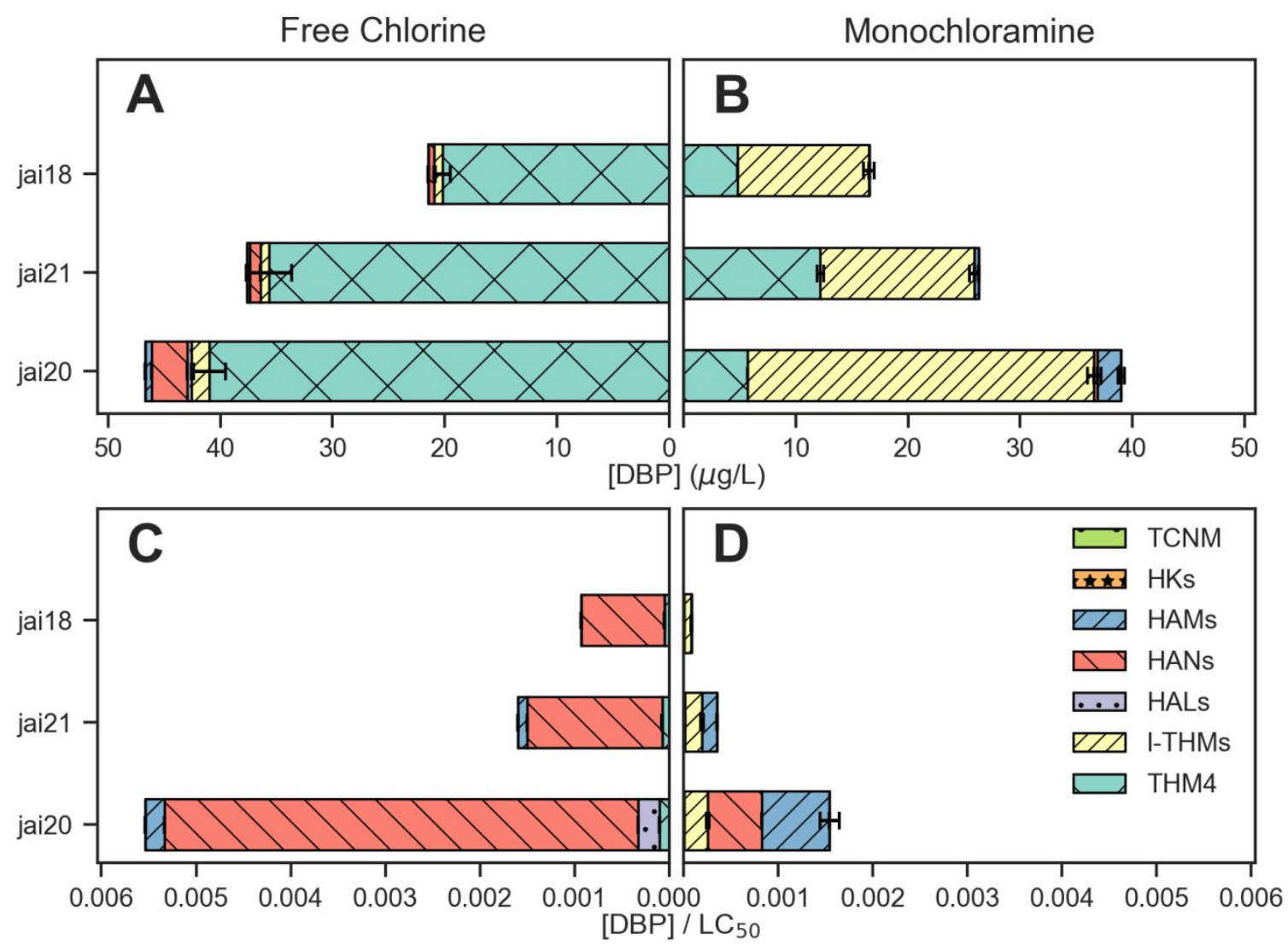

Figure S5: DBP concentrations by weight $(A, B)$ or by toxic potency $(C, D)$ in Jaipur groundwaters treated by free chlorine $(\mathrm{A}, \mathrm{C})$ or preformed monochloramine $(\mathrm{B}, \mathrm{D})$ with target residuals of $1 \mathrm{mg}-\mathrm{Cl}_{2} / \mathrm{L}$. 


\section{References}

Indian Standard Drinking Water Specification (Second Revision). Bureau of Indian Standards, IS 10500 (May), 1-11, 2012; http://cgwb.gov.in/Documents/WQ-standards.pdf (retrieved June 5, 2019).

Bond, T.; Templeton, M. R.; Mokhtar Kamal, N. H.; Graham, N., Kanda, R; Nitrogenous disinfection byproducts in English drinking water supply systems: Occurrence, bromine substitution and correlation analysis. Water Res., 2015, 85, 85-94. https://doi.org/10.1016/j.watres.2015.08.015

Chuang, Y.-H.; Szczuka, A.; Mitch, W. A. Comparison of Toxicity-Weighted Disinfection Byproduct Concentrations in Potable Reuse Waters to Conventional Drinking Waters as a New Approach to Assess the Quality of Advanced Treatment Train Waters. Environ. Sci. Technol. 2019, 53, 3729-3738.

Furst, K. E.; Pecson, B. M.; Webber, B. D.; Mitch, W. A. Tradeoffs between Pathogen Inactivation and Disinfection Byproduct Formation during Sequential Chlorine and Chloramine Disinfection for Wastewater Reuse. Water Res. 2018, 143, 579-588.

ISIRI (Institute of Standards, Industrial Research of Iran). Physical and Chemical Quality of Drinking Water, No. 1053, 5th ed. ISIRI, Tehran, 2009.

Jeong, C. H.; Postigo, C.; Richardson, S. D.; Simmons, J. E.; Kimura, S. Y.; Marinas, B. J.; Barcelo, D.; Liang, P.; Wagner, E.D.; Plewa, M. J. Occurrence and Comparative Toxicity of Haloacetaldehyde Disinfection Byproducts in Drinking Water. Environ. Sci. \& Tech. 2015, 49(23), 13749-13759. https://doi.org/10.1021/es506358x

Krasner, S. W.; Weinberg, H. S.; Richardson, S. D.; Pastor, S. J.; Chinn, R.; Sclimenti, M. J.; Onstad, G. D.; Thruston, A. D. Occurrence of a New Generation of Disinfection Byproducts. Environ. Sci. Technol. 2006, 40, 7175-7185.

Krasner, S. W.; McGuire, M. J.; Jacangelo, J. G.; Patania, N. L.; Reagan, K. M.; Aieta, E. M. The Occurrence of Disinfection By-Products in US Drinking Water. J. Am. Water Works Assoc. 1989, 81 (8), 41-53.

Kumari, M.; Gupta, S. K.; Mishra, B. K. Multi-exposure cancer and non-cancer risk assessment of trihalomethanes in drinking water supplies - A case study of Eastern region of India. Ecotoxicology and Environmental Safety, 2015, 113, 433-438.

Ministry of Health of Egypt. Criteria for Potable Water. Cairo, 2007.

Ministry of Health of People's Republic of China. Standards for Drinking Water Quality (GB 57492006). 2006, 12.

Mishra, B.; Gupta, S.; Sinha, A. Human Health Risk Analysis from Disinfection By-Products (DBPs) in Drinking and Bathing Water of Some Indian Cities. J. Environ. Heal. Sci. Eng. 2014, 12 (1), 73. https://doi.org/10.1186/2052-336X-12-73.

MNWL, Mexican National Water Law, Norm NOM-127-SSA1-1994, 1994. 
Wagner, E. D.; Plewa, M. J. CHO cell cytotoxicity and genotoxicity analyses of disinfection by-products: An updated review. J. Environ. Sci., 2017, 58, 64-76.

Wei, J.; Ye, B.; Wang, W.; Yang, L.; Tao, J.; Hang, Z. Spatial and temporal evaluations of disinfection by-products in drinking water distribution systems in Beijing, China. Sci. Tot. Environ. 2010, 408(20), 4600-4606. https://doi.org/10.1016/j.scitotenv.2010.06.053

Weinberg, H. S.; Krasner, S. W.; Richardson, S. D.; Thruston, A. D. The Occurrence of Disinfection ByProducts (DBPs) of Health Concern in Drinking Water: Results of a Nationwide DBP Occurrence Study; Athens, GA, 2002. https://doi.org/EPA/600/R-02/068. (retrieved June 5, 2019).

Williams, D. T.; LeBel, G. L.; Benoit, F. M. Disinfection By-Products in Canadian Drinking Water. Chemosphere 1997, 34, 299-316.

World Health Organization. Halogenated Acetonitriles in Drinking-water; Geneva, 2004. Retrieved from http://www.who.int/water_sanitation_health/dwq/chemicals/halogenatedacetonit.pdf

World Health Organization. Trihalomethanes in Drinking Water. Background Document for Development of WHO Guidelines for Drinking-Water Quality; Geneva, 2005; Vol. 67; http://www.who.int/water_sanitation_health/dwq/chemicals/THM200605.pdf

World Health Organization. Guidelines for Drinking Water Quality, 4th ed.; Geneva, 2011; https://doi.org/10.1016/S1462-0758(00)00006-6

Zeng, T.; Plewa, M. J.; Mitch, W. A. N-Nitrosamines and Halogenated Disinfection Byproducts in U.S. Full Advanced Treatment Trains for Potable Reuse. Water Res. 2016, 101, 176-186..

US EPA. ICR Auxilliary 1 Database Version 4 [Microsoft Access File] 2000. https://www.epa.gov/dwsixyearreview/supplemental-data-six-year-review-3 (retrieved Aug. 8, 2019)

U.S. Environmental Protection Agency. Fed. Regist. 1998, 63, 69390-69476.

U.S. Environmental Protection Agency. Office of Research and Development (ORD), National Center for Environmental Assessment. Integrated Risk Information System. https://www.epa.gov/iris (retrieved May 24, 2019). 